\title{
Single-Incision Laparoscopic Multivisceral Resection
}

\author{
Min Li, MM, Shuodong Wu, MD, Yu Tian, MD, Yongnan Li, MM, Hong Yu, MD, \\ Yongsheng Chen, MM \\ Department of Biliary and Vascular Surgery, Shengjing Hospital of China Medical University, Shenyang, China \\ (all authors).
}

\begin{abstract}
Introduction: Our aim was to report a case regarding combined resection of a hepatocellular carcinoma, hepatic cyst, gallbladder, and appendix.

Case Description: The patient underwent single-incision laparoscopic surgery for resection of a hepatocellular carcinoma, hepatic cyst, gallbladder and appendix.

Discussion: There was $<200 \mathrm{~mL}$ of blood loss without transfusion. The operation took 140 minutes. The patient's bowel function recovered on day 3, and he was discharged on day 9. When assessed 1 year after the operation, there was no recurrence of the tumor. Single-incision laparoscopic multiple-organ resection is a safe and feasible option for selected patients, which is worth exploring in clinical work.
\end{abstract}

Key Words: Single-incision laparoscopic surgery, Multivisceral resection.

Citation Li M, Wu S, Tian Y, Li Y, Yu H, Chen Y. Single-incision laparoscopic multivisceral resection: report of a case. CRSLS e2013.00221. DOI: 10.4293/CRSLS.2013.00221.

Copyright ( 2015 by SLS, Society of Laparoendoscopic Surgeons. This is an open-access article distributed under the terms of the Creative Commons Attribution-Noncommercial-ShareAlike 3.0 Unported license, which permits unrestricted noncommercial use, distribution, and reproduction in any medium, provided the original author and source are credited.

Address correspondence to: Shuodong Wu, Department of Biliary and Vascular Surgery, Shengjing Hospital of China Medical University, Shenyang, Liaoning Province, 110004, China. Telephone: +86-024-96615-1-31211, Fax: +86-24-31803141, E-mail: wushuodong@yahoo.cn

\section{INTRODUCTION}

Laparoscopic multiorgan resection is a surgical procedure in which $\geq 2$ organs are managed in 1 operation. Laparoscopic surgery is likely to benefit patients by reducing physiological and psychological stress related to reoperation, and it is considered to offer better cosmesis. ${ }^{1}$ With the development of laparoscopic technique, transumbilical single-incision laparoscopic surgery (SILS) has attracted more and more surgeons in recent years because of its minimal invasiveness and better cosmetic results with fewer skin incisions. Our department has completed a series of operations using a single-incision laparoscopic technique. We report a case of combined resection of a hepatocellular carcinoma, hepatic cyst, gallbladder, and appendix.

\section{Case Description}

A 64-year-old man was admitted to the hospital with a 1-year history of epigastric discomfort and pain. He had repeated episodes of cholecystitis and appendicitis. On physical examination, the abdomen was tender with no palpable masses and the Murphy sign was positive. Laboratory tests showed that the patient tested positive for hepatitis B surface antigen and the alpha fetoprotein level was $9.72 \mathrm{ng} / \mathrm{mL}$ (normal range, $0-9 \mathrm{ng} / \mathrm{mL}$ ). The computed tomography scan with enhancement (Figure 1a) showed a mass on segment 8 of the liver, which could not be distinguished as a benign or malignant lesion. From the computed tomography scan (Figures 1b, 1c, and 1d), we also found multiple intrahepatic cysts, a gallbladder stone, and a small fecalith in the appendix. The preoperative diagnosis was hepatocellular carcinoma (not confirmed), hepatic cysts, a gallbladder stone, and appendicitis.

The patient was placed in the supine position under general anesthesia. After pneumoperitoneum $(13 \mathrm{~mm} \mathrm{Hg}$ ) was induced $(1 \mathrm{~mm} \mathrm{Hg}=0.133 \mathrm{kPa})$, the patient was moved into the Trendelenburg position with his left side lower for better exposure (Figure 2a). A 2.5-cm longitudinal incision was made through the umbilicus, and 3 trocars (one 5-mm and two 10-mm trocars, which are traditional laparoscopic instruments) (Figure 3a) were inserted in the shape of an inverted triangle (Figure $3 \mathbf{b}$ ). One of the 10-mm 


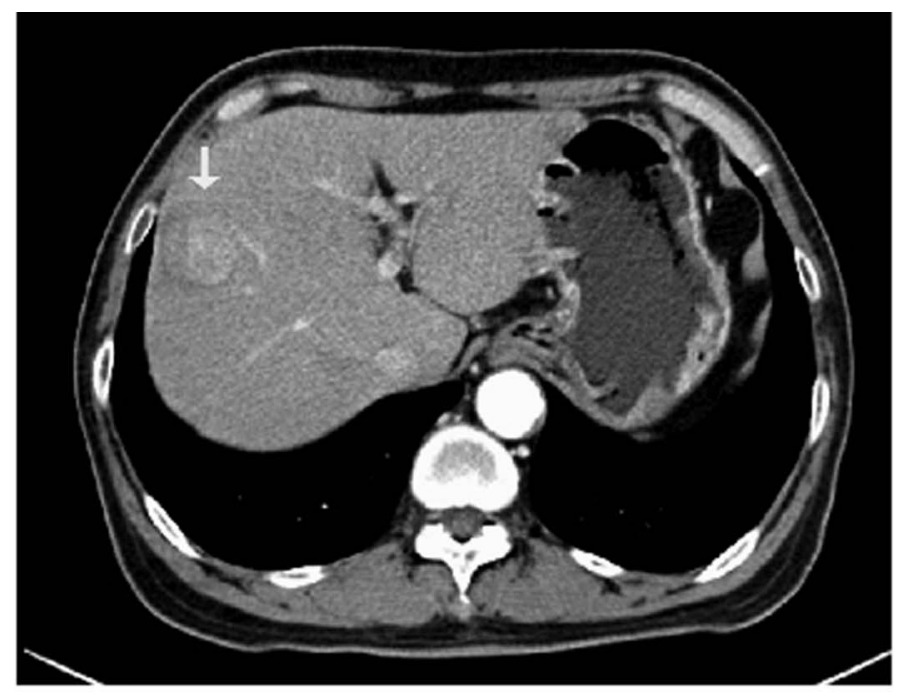

a

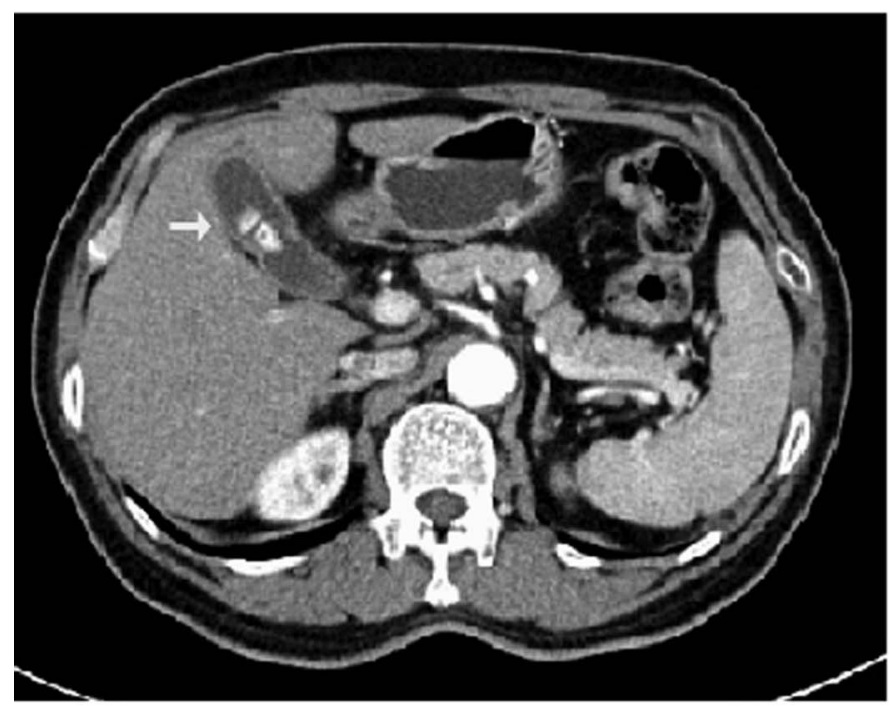

C

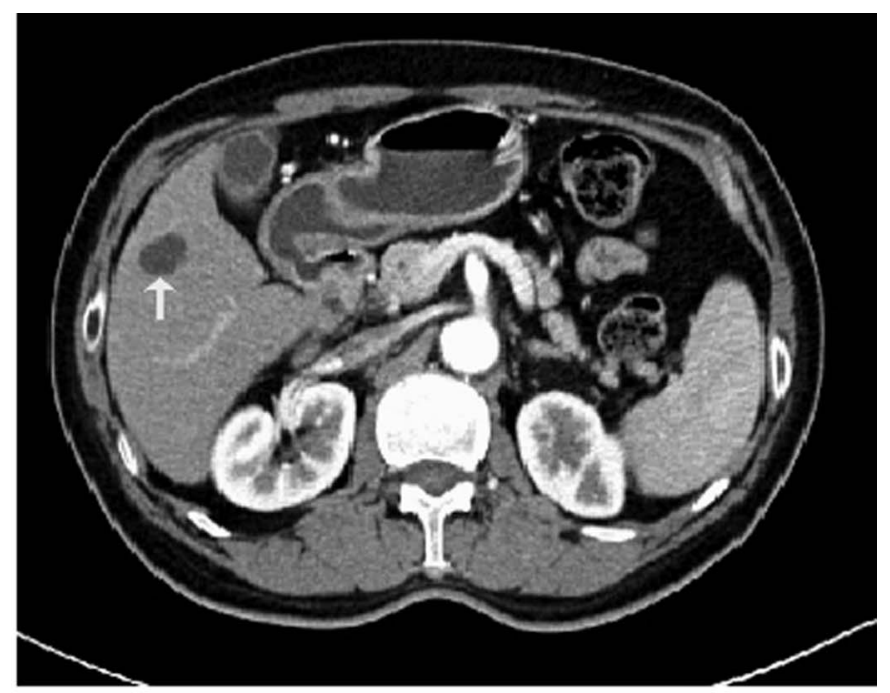

b

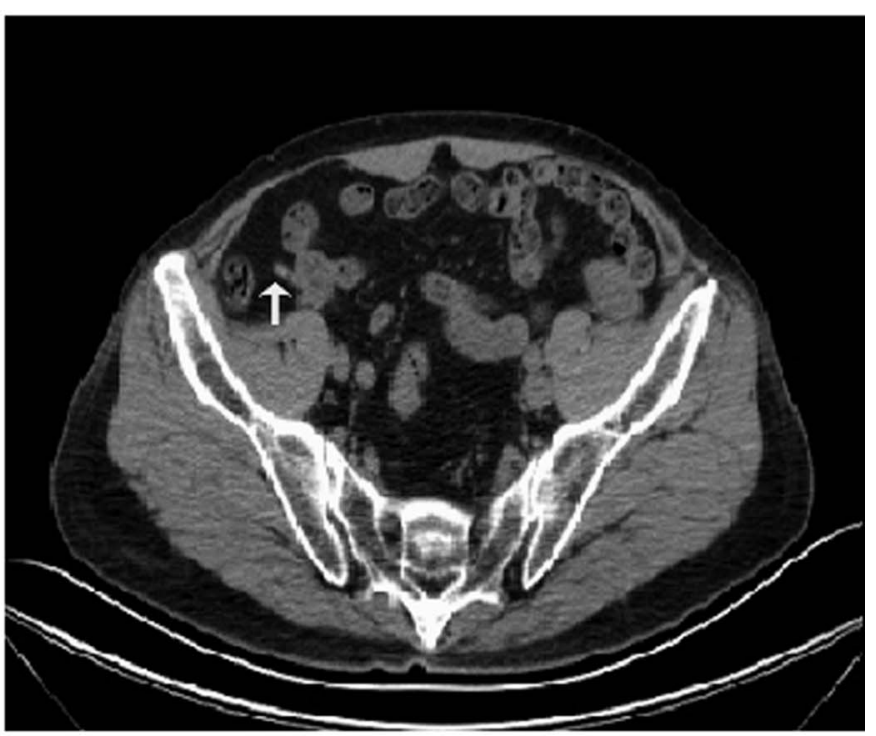

d

Figure 1. (a) Mass on segment 8 of liver (arrow). (b) Three-centimeter cyst found on liver (arrow). (c) Gallbladder stone (arrow). (d) Small fecalith in appendix (arrow).

trocars was used for the rigid $30^{\circ}$ laparoscopic lens, and the other was used for the primary manipulation.

First, the operator used a Harmonic (LCS; Ethicon, Cincinnati, Ohio) scalpel to divide the mesentery of the appendix (Figure 4a). Then, a Hemolock (Teleflex Medical Europe Ltd., Athlone Co., Westmeath, Ireland) clip was used on the root of the appendix. After that, the appendix was transected and removed from the 10-mm trocar. Next, the patient was moved into the reverse Trendelenburg position with his left side lower, the surgeon and assistant exchanged their positions, and the monitor was placed on the right shoulder side of the patient (Figure $\mathbf{2 b}$ ). The gallbladder was dissected from the liver with the Harmonic scalpel, and the Calot triangle was dissected to expose the cystic duct (Figure $\mathbf{4 b}$ ). After the cystic dust was closed with 2 Hemolock clips, the gallbladder was transected and removed. A 3-cm liver cyst was found on the liver surface (Figure 4c). We aspirated the cyst and found that the content was clear without bile or parasites. Part of the cyst wall was removed by application 

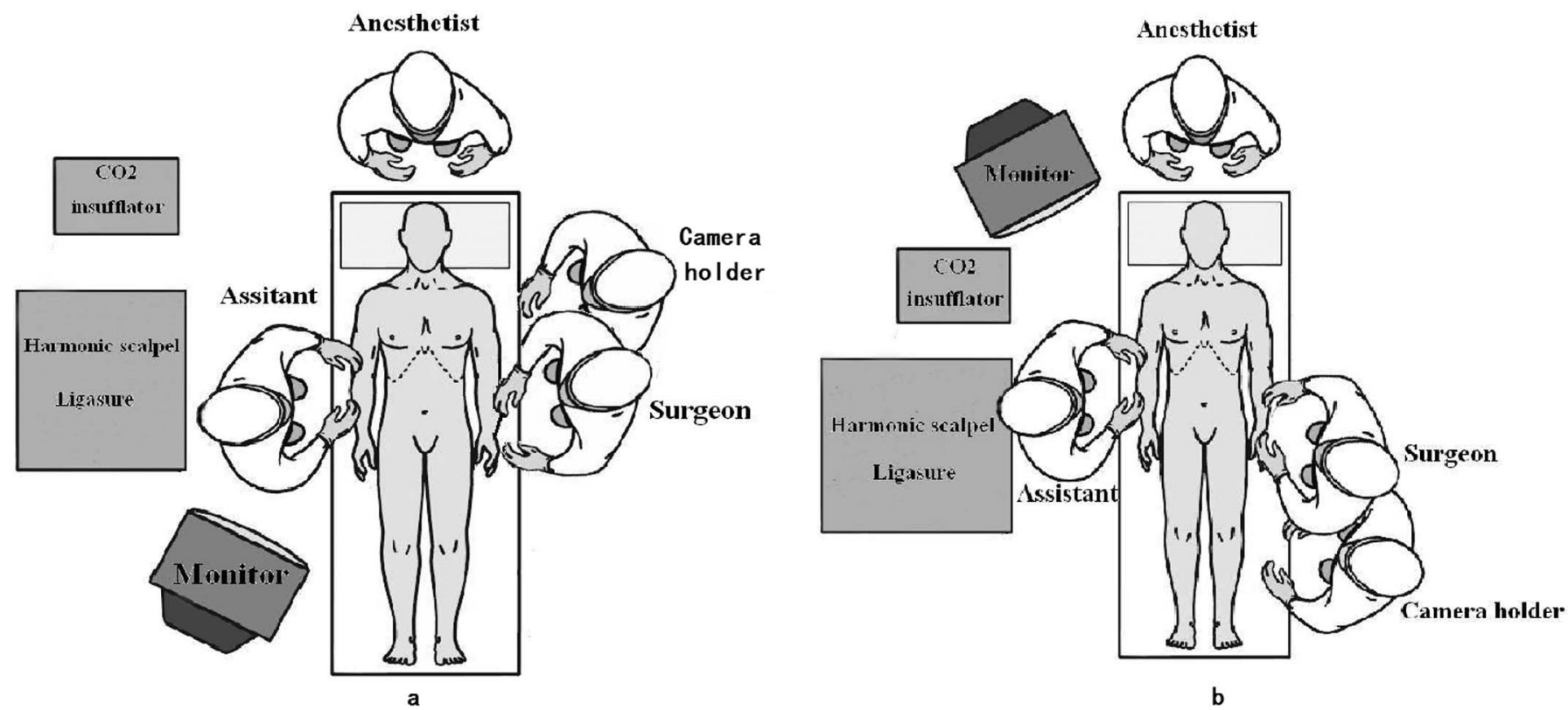

Figure 2. (a) Position for appendectomy. (b) Position for other operations.

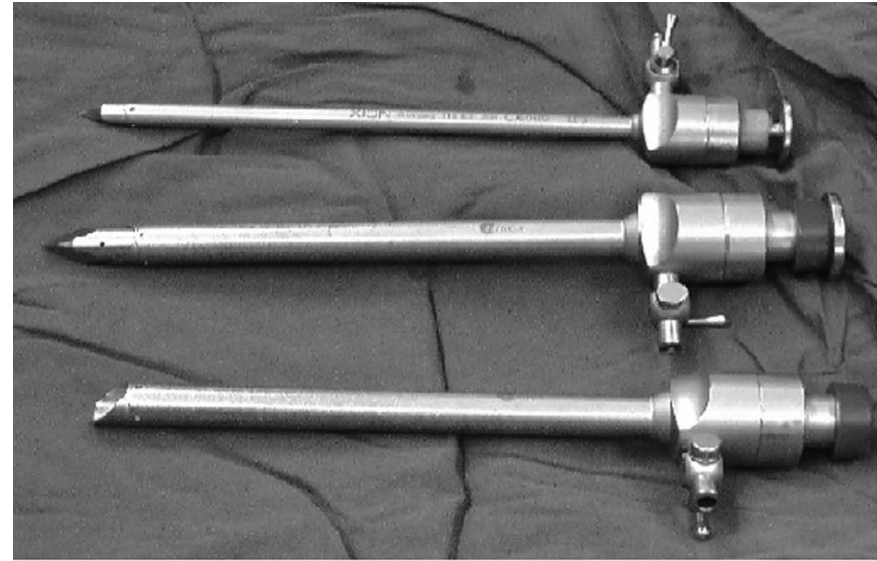

a

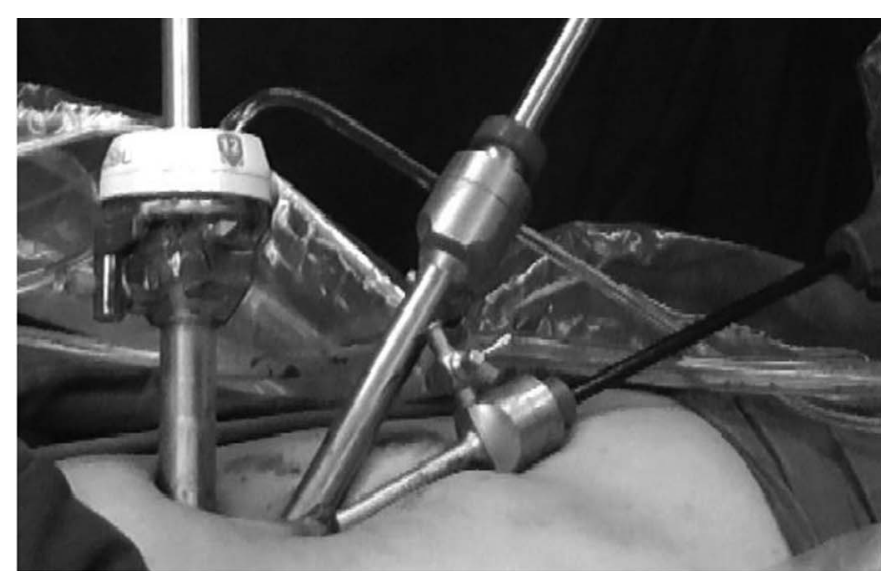

b

Figure 3. (a) Position of 3 trocars. (b) Trocars used during surgery.

of the Harmonic scalpel. The hepatoduodenal ligament was dissected, and a 10-French urethral catheter was present in case a Pringle block occurred during liver resection. The tumor of the liver was located on segment 8 of the liver surface. A 2 -cm radius of normal liver tissue around the tumor was marked by an electrocautery hook to define the resection line. The tumor was removed along with some normal liver tissue in a wedge shape by a combination of Ligasure (Tyco Healthcare Group, LP Boulder, Colorado) and the Harmonic scalpel (Figure 4d). Pringle occlusion was not performed throughout the operation because of slight blood loss. The gallbladder, wall of the cyst, and tumor were packed in an aseptic specimen bag and removed from the umbilical incision (Figure 5a). After careful assessment, drainage tubes were placed around both the right subdiaphragm and Winslow window and extruded out of the abdomen through the umbilicus (Figure 5b).

There was $<200 \mathrm{~mL}$ of blood loss without transfusion. The operation took 140 minutes. The liver tumor was proved to be hepatic cellular cancer after pathologic examination (Figure 5c). The patient's bowel function recovered on day 3 , and he was discharged on day 9. 


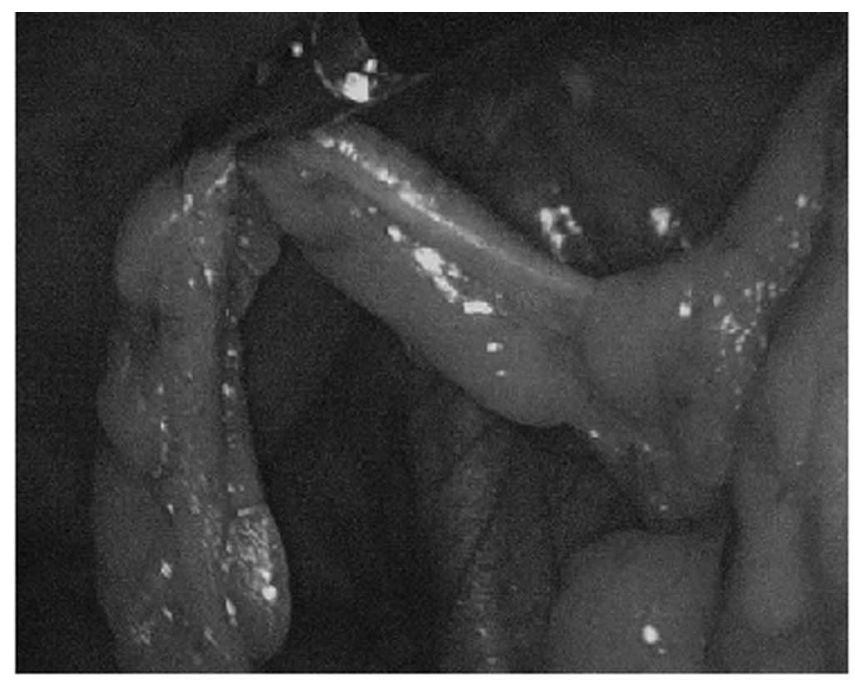

a

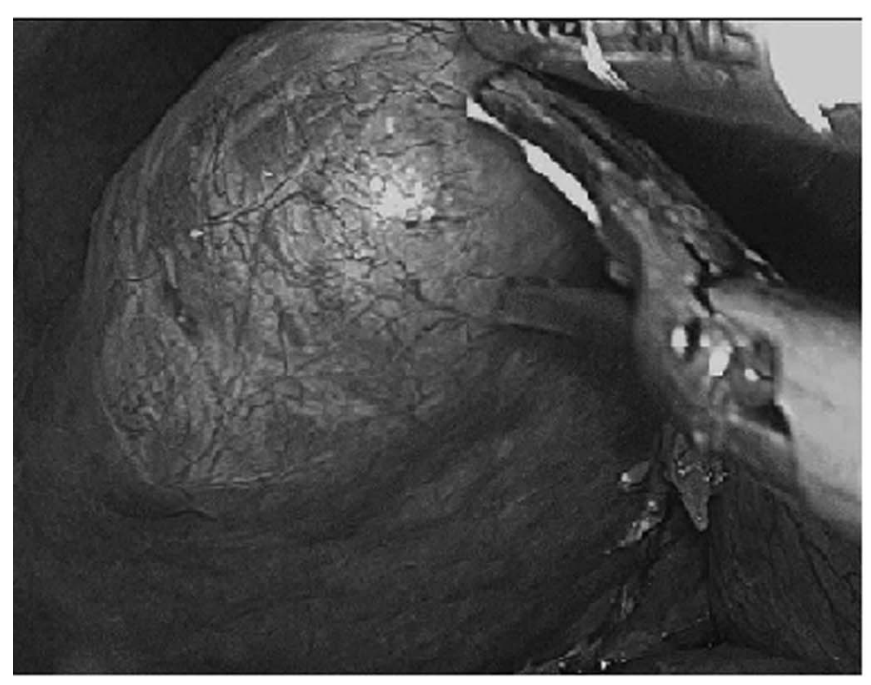

c

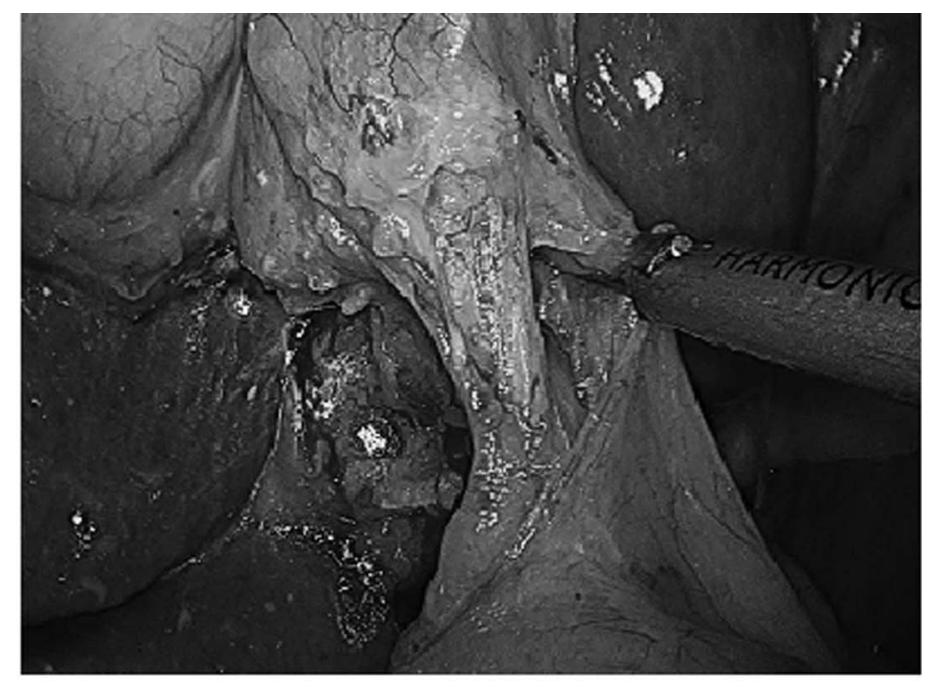

b

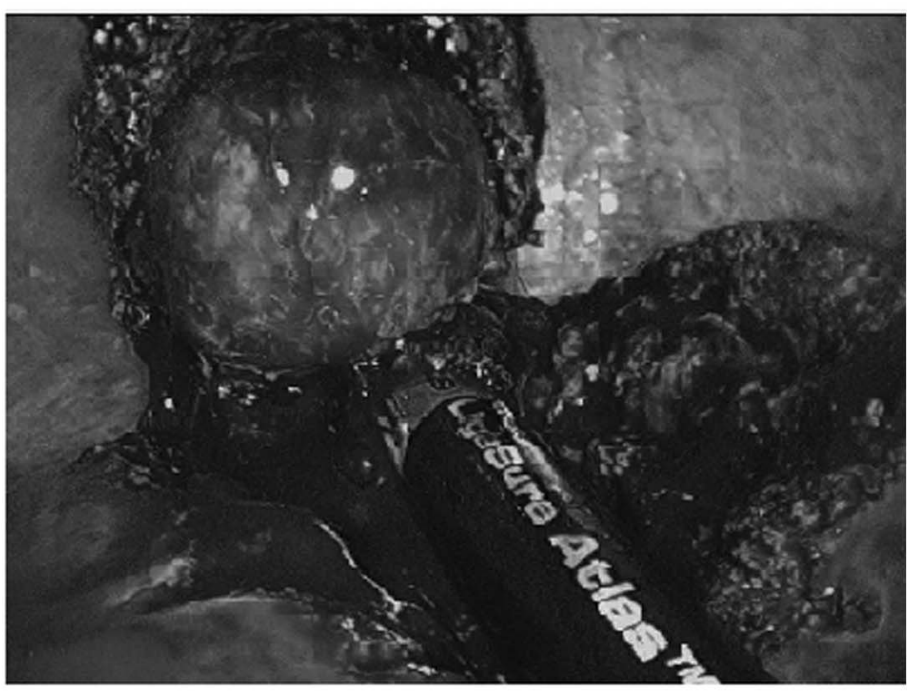

d

Figure 4. (a) Exposure of appendix. (b) Exposure of Calot triangle. (c) Three-centimeter cyst found on liver surface. (d) The tumor was located on segment 8 of the liver surface and was removed by a combination of Ligasure and the Harmonic scalpel.

When assessed 1 year after the operation, there was no recurrence of the tumor.

\section{DISCUSSION}

Laparoscopic surgery has brought about changes in general surgery. It is characterized as safe, minimally invasive, and more esthetically pleasant with a shorter hospital stay. ${ }^{2,3}$ Multivisceral resection is defined as surgery in which $\geq 2$ organs are managed in 1 operation. However, in multiple-incision laparoscopic surgery, well-planned positioning of trocars is another challenge for surgeons. It may be inconvenient because of compromising insertion locations, which may decrease the efficacy of the operation. SILS can be performed on various organs in the entire abdominal cavity at the same time through position exchange of the surgeon and his or her assistants and by switching between 5-mm and 10-mm laparoscopic lens. Vidal et $\mathrm{al}^{4}$ compared SILS for appendectomy with a 3-site technique and believed that there was no difference between groups in terms of recovery and hospital stay. Cai et $\mathrm{al}^{5}$ also thought that although SILS was more difficult to perform, it was safer and more feasible, minimally inva- 


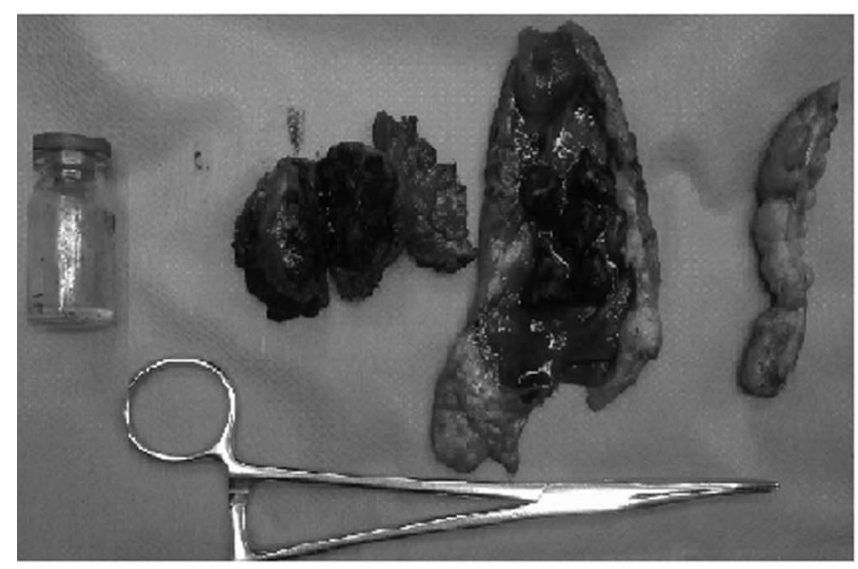

a

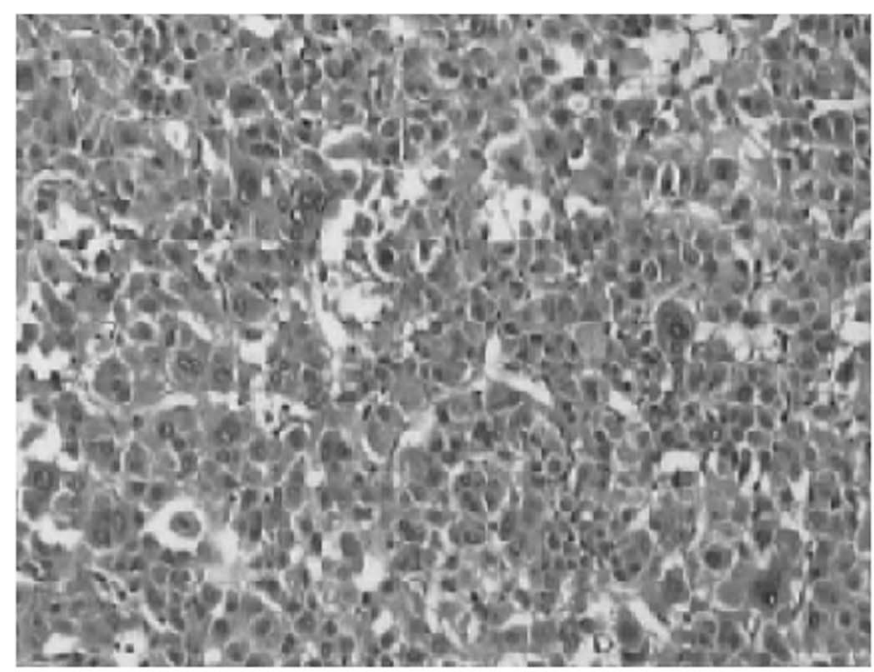

C

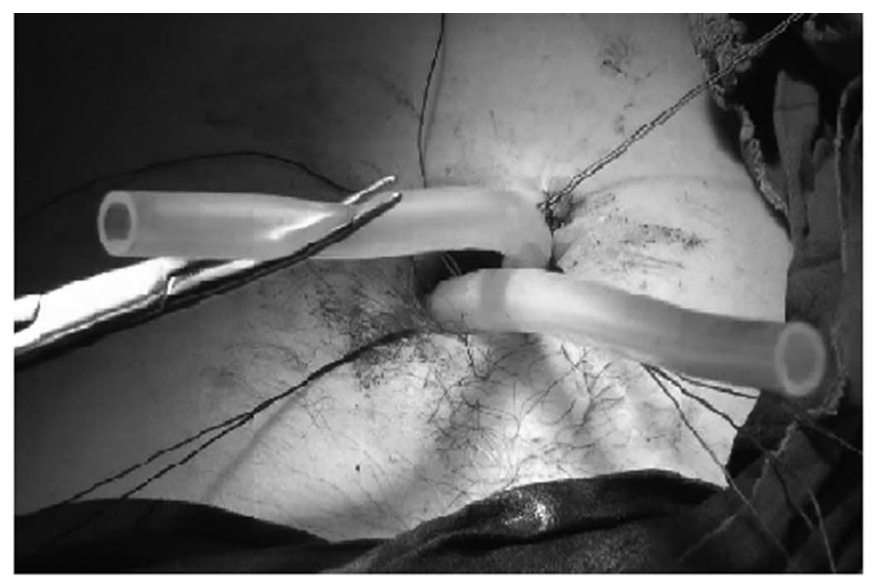

b

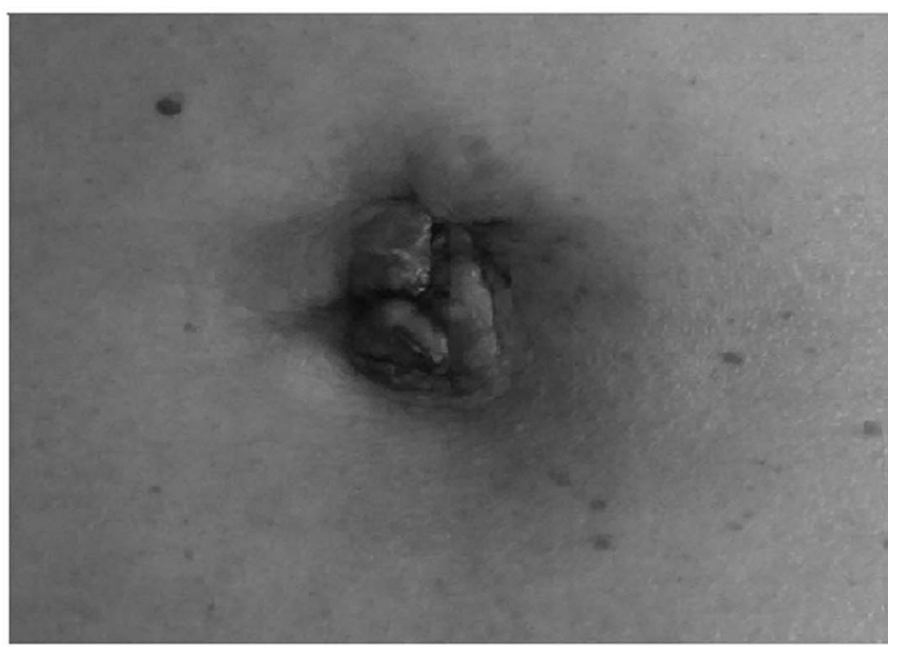

d

Figure 5. (a) Specimens: wall of cyst, tumor, gallbladder, and appendix. (b) Drainage tubes extruded out of the abdomen through the umbilicus. (c) The tumor was proved to be hepatic cellular cancer after pathologic examination. (d) The umbilical incision was sutured with polygalctin 910 suture, and there would be no scar in the rest of the abdominal wall.

sive, and cosmetic. Drainage could be taken out through the umbilical incision. After removal of drainage, the umbilical incision is sutured with polyglactin 910 suture, and there would be no scar in the rest of the abdominal wall (Figure 5d).

Careful selection of patients is of vital importance in single-incision laparoscopic multivisceral resection. Because of $\mathrm{CO}_{2}$ pneumoperitoneum, a higher demand of cardiopulmonary function in patients is necessary. Besides, multivisceral laparoscopic surgery takes longer than singleorgan surgery, which inevitably leads to a higher risk of anethesia. Accordingly, we should perform a thorough medical examination of patients and improve their body condition before performing the operation. Furthermore, we must know the indications well and have a clear head to solve major problems. It is forbidden to blindly broaden the surgical indications to increase the number of multiple-organ resections. In this case the diameter of the hepatic cyst was $3 \mathrm{~cm}$; we resected it because it was combined with other epigastric surgical procedures and was located close to the surface. Before carrying out SILS, less experienced surgeons should select patients without a history of abdominal operations.

In addition to a surgeon's rich experience performing laparoscopic surgery, single-incision multivisceral laparoscopic surgery requires favorable cooperation between 
the operator and assistant. A coaxial effect will be created just like the use of chopsticks because all devices are placed through the same umbilical incision during surgery. Using regular laparoscopic instruments rather than the Tri-Port system (Covidien, USA) increases difficulties because the instruments are very close together and interfere with each other. During the operation, we follow the principle of "only move two every time"- that is, when the operator is manipulating with both hands under the vision device, the laparoscopic lens should be kept still, and when the surgeon is performing a detailed operation with his or her left hand immobile, the assistant should adjust the distance and direction of the laparoscopic lens to improve visualization.

\section{CONCLUSION}

The patient had not only a hepatic cellular carcinoma but also a hepatic cyst, cholecystolithiasis, and chronic appendicitis. The traditional open operation has many problems, such as a long incision, maximal invasiveness, large amount of blood loss, and complications, that could be avoided by use of single-incision surgery. The major advantage of SILS over standard laparoscopic surgery is the cosmetic result, which is worth exploring in clinical work.

\section{References:}

1. Kim HJ, Chhol GS, Park JS, et al. Simultaneous laparoscopic multi-organ resection combined with colorectal cancer: comparison with non-combined surgery. World J Gastroenterol. 2012; 18(8):806-813.

2. Kim KY, Hwang DW, Park YK, et al. A single surgeon's experience with 54 consecutive cases of multivisceral resection for locally advanced primary colorectal cancer: can the laparoscopic approach be performed safely? Surg Endosc. 2012;26(2): 493-500.

3. Hemandas AK, Abdelrahman T, Flashman KG, et al. Laparoscopic colorectal surgery produces better outcomes for high risk cancer patients compared to open surgery. Ann Surg. 2010; 252(1):84-89.

4. Vidal O, Valentini M, Ginesta C, et al. Laparoendoscopic singlesite surgery appendectomy. Surg Endosc. 2010;24(3):686-691.

5. Cai W, Xu J, Zheng M, et al. Combined laparoendoscopic single-site surgery: initial experience of a single center. Hepatogastroenterology. 2012;59(116):986-989. 